\title{
Ensino e Aprendizagem de Matemática na Engenharia e o Uso de Tecnologia*
}

Tânia Cristina Baptista Cabral**

Resumo: Neste trabalho trazemos para o debate concepções e questões sobre a função e o uso de computadores no ensino e na aprendizagem das matemáticas em um curso de engenharia, em modo presencial. Apresentamos: (1) concepções sobre a relação entre tecnologia, educação matemática e educação em engenharia; (2) concepções para o ensino e a aprendizagem em um curso de engenharia específico e (3) uma proposta de ambiente.

Palavras-chaves: Educação Matemática e Engenharia, Ambiente Interativo e Multidisciplinar; Ensino e Aprendizagem de Cálculo.

Abstract: In this paper we discuss conceptions about the use of computers in teaching and learning of mathematics in engineering courses. We present: (1) conceptions about the relation between technology, mathematics education and engineering education; (2) teaching and learning conceptions in a specific engineering course and (3) a proposal of a technological teaching and learning environment.

Key-words: Mathematics Education and Engineering; Multidisciplinary and Interactive Environment; Teaching and Learning of Calculus.

\section{Introdução: Discutindo Tecnologia, Educação Matemática e Educação em Engenharia}

A época atual é de forte apelo tecnológico, de transmissão de dados em alta velocidade e de troca de informações em tempo real. Pensar na educação tecnológica nos leva a refletir sobre como estabelecer novos parâmetros educacionais visando modificações no trabalho de formulação de atividades didáticas que possam ser associadas ao uso de computadores ou de qualquer outra mídia "If the media change, paper and pencil to a plotter, for instance, the manner of teaching the concept of function, for example, will change" (Borba, v.2, p. 172, 2005). Também é exigido um olhar diferente sobre a significação que damos a sujeitos aprendentes. Como um aluno responde às inserções de aparato tecnológico? Que demandas lhe são postas?

O papel da tecnologia em sala de aula exige novas formas de se conceber o ensino e aprendizagem de matemática "If mathematics is seen as a rigid body of unquestionable truths, then there is no possibility of discussing the influence of media in the mathematics produced by students" (Borba and Villarreal, p. 197, 2005) e realizar estudos acerca das consequiências de se adotar softwares, sistemas computacionais e similares

What perspectives are used to investigate the use of technology in Mathematics Education in different countries? 2. How would new perspectives allow us to re/think the role of users of technology? 3. What new methodologies would enable us to investigate difficult issues concerning teaching and learning situations in micro-world environment? (Lins, Giraldi et al.: v.1 p.265, 2004)

$\mathrm{Na}$ mesma problemática, há reflexões sobre o efeito de se conceber o trabalho com computadores restrito a laboratórios específicos, servindo apenas para reafirmar o que foi ensinado em sala conduzida por metodologia de ensino expositiva: "Muitas vezes, os professores, premidos pelas exigências oficiais e institucionais, levam seus

\footnotetext{
*Apoio: FAPERGS.

Professora Doutora, pesquisadora pela FAPERGS no curso de Engenharia em Sistemas Digitais, Unidade de Guaíba, UERGS. tania.c.b.cabral@terra.com.br. Grupo de pesquisa: www.gritee.com. 
alunos a laboratórios de informática sem planejar que desenvolvem competências, tais como a de formular hipóteses ou de argumentar" (Cury, p. 19, 2004). Nesse mesmo movimento, conceitos estão sendo revisitados. A exemplo, no desenvolvimento de sistemas inteligentes, há posições que admitem que a linguagem tem o papel expressar o mundo ou objetos a fim de projetar recursos que viabilizem e tornem claros conhecimentos que não seriam compreendidos pela máquina - pensando-se em termos de inteligência artificial ou de objetos dirigidos a agentes artificiais - através de verbalização. A linguagem abrange várias feições tais como imagem, figura, diagramas e desenhos para tentar externar conhecimento que deverá ser processado, transmitido e reconhecido "Linguagens diagramáticas podem dar ao usuário uma alternativa para visualizar os problemas do mundo real e manipular seus elementos por meio de gráficos" (Garcia, Varejão e Ferraz, p. 65, 2003). Na base dessa concepção está a estrutura agente emissor, agente receptor e mensagem, foco de teorias lingüísticas e de tratamentos dados à comunicação em domínios que envolvem o ensino e aprendizagem através, por exemplo, do uso de computadores. Trata-se de um ponto polêmico, pois, pensado em termos discursivos, é atribuído ao agente emissor a responsabilidade pela significação do que é dito e do que é realizado.

Como contra ponto, as relações sócio-cognitivas imbricadas no cenário da sala de aula indicam que o discurso não pode ser entendido ou reduzido a uma cadeia de palavras ou sinais (desenho, figuras, etc.) emitidos por um sujeito e ouvidos ou lidos por outro. Na situação que envolve o ensino, quando se presta atenção às reações do aluno e às significações produzidas no entorno onde ocorrem processos de aprendizagem, observa-se que o fenômeno da fala é muito mais complexo porque envolve necessariamente um desentendimento estrutural.

A posição epistemológica a partir da qual vimos investigando os fenômenos de aprendizagem permite conceituarmos as formas discursivas como sendo o estatuto das declarações que requer escolhas entre possíveis falas em acordo com direções que interlocutores, engajados em uma situação, esforçam-se em manter. Assim, a comunicação (ou ato comunicativo como também é designado em estudos na área da inteligência artificial) é entendida como um desentendimento que foi bem sucedido: o discurso é um esforço conjunto de sujeitos - alunos e professor no caso da situação de sala de aula - para sustentar uma particular relação de ações e declarações. Quando falamos nos subjetivamos em um processo intrincado por abarcar pré-suposições de uma ação, ao menos uma estrutura de linguagem simbólica e tudo que diz respeito a nossas crenças, escolhas, intenções, dúvidas e gostos.

O uso do computador no trabalho da docência, presencial ou a distância, não pode ser considerado mais um mero dispositivo didático complementar e meramente técnico que possibilite condições de efetivação de comunicação entre alunos e professor "Tools cannot be though of as external facilitators of a process of learning, which, would proceed towards pre-established aims anyway" (Borba and Villarreal, p. 213, 2005). Assim como não podemos ignorar os efeitos indiretos provocados pelo trabalho realizado na educação a distância "No contexto das Engenharias, várias experiências já foram realizadas e os resultados obtidos nos permitem afirmar que a EAD propicia o repensar do ensino presencial" (Flemming, p. 285, 2004). Delineia-se a necessidade de se ter clareza sobre a movimentação do campo da produção de conhecimento para aceitar novos pressupostos que sejam convenientes ao trabalho em desenvolvimento, de modo que nem impeçam o avanço puramente técnico de inovar e tampouco destruam o que já foi fundado em termos pedagógicos e didáticos. 
Estas questões incitam-nos a indagar sobre que objetos matemáticos devem ser trabalhados e, ao mesmo, tempo, demandam termos de repensar o tratamento a ser dado a estes objetos. Que epistemologia deve sustentar o trabalho e que formas podem ser criadas para organizar uma turma e avaliar o aluno? Objetivando a inclusão digital, consideradas a via da educação presencial e via da educação a distância, formula-se uma base de estudos sobre fatores sociais e psicológicos. O reconhecimento da existência de dificuldades em torno do ato de ensinar matemática bem como a admissão que se lida com cognição e afetividade, aspectos constituintes da subjetividade, delineiam um ponto de partida para projetar modificações metodológicas.

\section{Concepções para o ensino e a aprendizagem em curso de engenharia}

Conforme a discussão feita por Costa (2004) os ambientes tecnológicos alteraram o modo de se resolver problemas. Uma vez modificados os processos de modelagem e resolução, vemos implicações diretas na construção dos conceitos que determinam as ferramentas a serem usadas nesses processos. Isso ocorre também na educação a distância que sofre alterações a partir da melhoria de ambientes tecnológicos quando são agregados recursos que, síncronos ou assíncronos, permitem o contato virtual - a conversa não presencial.

Em uma unidade universitária, distinções no modo de conceber o curso e seus objetivos, no que diz respeito aos condicionantes institucionais, podem impor condições claras sobre o trabalho que, de modo coordenado por uma equipe de professores do curso, tem de ser desenvolvido em algumas disciplinas. Visando o ensino e aprendizagem presencial de matemática em um curso de engenharia com grande heterogeneidade de alunos decorrente de uma política de reserva de vagas adotada pela universidade, é preciso conciliar aspectos relativos à didática, pedagogia, epistemologia e tecnologia. Uma resposta é a integração de disciplinas matemáticas - afinadas com outras disciplinas ditas de engenharia, como Laboratório de Circuitos Elétricos ou Lógica - em ambientes que possam ser estruturados através de sistemas computacionais inteligentes. Mas, para isso é preciso conceber as condições para a realização de um trabalho de aprendizagem cooperativo, interativo e multidisciplinar que propiciem ao aluno lidar com objeto-matemático profissional responsável pela sustentação da produção sócio-cultural em uma instituição de ensino superior.

\subsection{O trabalho de aprendizagem cooperativo, interativo e produtivo}

Para propor um ambiente visando à cooperação e interatividade envolvendo agentes alunos, professor e computador é preciso exprimir que bases sustentam a construção do ambiente. Foi a partir da insatisfação com ambientes rígidos, onde não há diálogo - ou quando acontece, é totalmente controlado pelo professor - que foi assumida em aulas de matemática para um curso de engenharia a pedagogia da Assimilação Solidária (AS) cujos princípios guiam nossa prática educativa.

Vale a pena recordar aqui a trajetória trilhada até ser formulada a proposta da AS, para entender algumas das reformulações feitas. Teorias de aprendizagem - formuladas por Piaget, Bruner, Ausubel, Vigostsky - foram estudadas em conjugação com aspectos que cada uma delas proporciona para reflexão: reequilibração majorante, aprendizagem ativa, pontos de ancoragem, zona de desenvolvimento proximal, etc. Desses estudos caracterizados por serem situados no campo da cognição foi preciso passar aos estudos da teoria formulada por Pichon-Riviere (1988) que, no campo da psicanálise social, analisa os papéis dos sujeitos que contribuem ou não para a realização de tarefas através de técnicas de grupos operativos. A teoria aborda a aprendizagem individual como mudança do sujeito que enfrenta e supera as dificuldades ao se responsabilizar por 
aspectos na tarefa de aprendizagem grupal. Mais alguns passos dados na direção de interpretar certas atitudes de alunos, foram buscados subsídios na psicanálise de orientação Freudo-Lacaniana para sustentar as duas faces da mesma moeda: o ensino e a aprendizagem. Derivamos dos estudos que desejar ensinar é querer ouvir o aluno e interpretar suas angustias relativas a seu modo de lidar com o objeto matemático. Desejar aprender é responsabilidade de cada indivíduo que tenha assumido compromisso de pertença com o grupo cuja imagem escolheu para ser identificado; logo, para aprender é preciso falar (Cabral, 1998). É nesse ponto que estamos hoje. A pedagogia que se adota como apoio à didática, portanto, é caracterizada por promover trabalho em grupo, buscando reforçar a produção coletiva de conhecimento e de responsabilidade de cada participante, segundo o lema "ensina-se ouvindo, aprende-se falando".

Nos pequenos grupos são incentivadas: a coleta de dados, a troca de informações, a simbolização de uma concepção e a construção de argumentos. $O$ resultado é que alunos dedicam maior atenção às tarefas e como eles próprios declaram durante a avaliação coletiva $^{1}$ ao final de cada aula, o engajamento não lhes deixa perceberem o tempo passar. Cientes de que os alunos têm dificuldades diferentes, procuramos organizá-los em grupos segundo o critério de homogeneidade na tarefa e heterogeneidade na formação. Para a homogeneidade na tarefa, redobramos esforços para observar e interpretar, qualitativamente, como os alunos respondem, como pensam, que dificuldades e facilidades demonstram com objetos-matemáticos básicos. Um grupo heterogêneo é formado por alunos que têm crenças distintas sobre vários assuntos do cotidiano como política, futebol, religião, economia - temas que costumam levantar polêmicas - e têm gostos diferentes para leitura, música, cinema, cultura.

A definição de pedagogia adotada para as aulas regulares gerou idéias e fundamentos para a construção de um ambiente tecnológico destinado à educação presencial. Pensar em diversos aspectos sociais, cognitivos e psicológicos, fatores que subjetivam as posições ditas sociais, impõe interpretar quais são as condições de engajamento dos alunos nas atividades propostas. As implicações daí decorrentes são contundentes porque exigem a busca de um conjunto de ações de negociação e de avaliação necessárias a, e compatíveis com, o desenvolvimento de um ambiente computacional que, de certo modo, possa fazer parte do conjunto de imagens que podem vir a ser escolhidas pelo aluno durante seu processo de aprendizagem. Portanto, a teoria de aprendizagem proposta suporta o trabalho de sala de aula através da cooperação, da interatividade e da produtividade contextualizada em situações onde imagens podem ser relacionadas pelo aluno com o ser engenheiro e, assim, promover a integração social.

Se a tecnologia em si tende a induzir o sujeito ao isolamento, o trabalho cooperativo, interativo e multidisciplinar, apoiado em oferta de imagens profissionais, pode resgatar o indivíduo e incluí-lo socialmente. Parece um paradoxo que a mesma idéia acerca de ambientes tecnológicos possa por um lado revelar a exclusão por falta de acesso assim como pode excluir quando não se tem interesse por estudo dos fatores de isolamento que os cercam.

\subsection{Trabalho cooperativo e o pensamento infinitesimal no cálculo do engenheiro}

\footnotetext{
${ }^{1}$ A cada aula, ao final, é realizada uma reunião plenária de 15 minutos, em média, onde a palavra é requisitada por qualquer um segundo a ordem de colocação. O objetivo da plenária é avaliar o trabalho do dia através dos aspectos didáticos, pedagógicos e epistemológicos. 
O desafio referente à didática recai sobre a escolha e abordagem de conteúdos de modo que as demandas de formação da engenharia sejam atendidas ao se apontar para a constituição de objetos-matemáticos profissionais. De fato, o que deve ser indicado é a natureza epistemológica do problema de legitimar um objeto de ensino, não a partir das garantias de exatidão e da organização vigentes na prática científica (da matemática), mas, sim, a partir da formação profissional, no caso a do engenheiro, conferindo-lhe uma outra unidade epistemológica. Dessa perspectiva, foi pressuposto que os estudos realizados nas

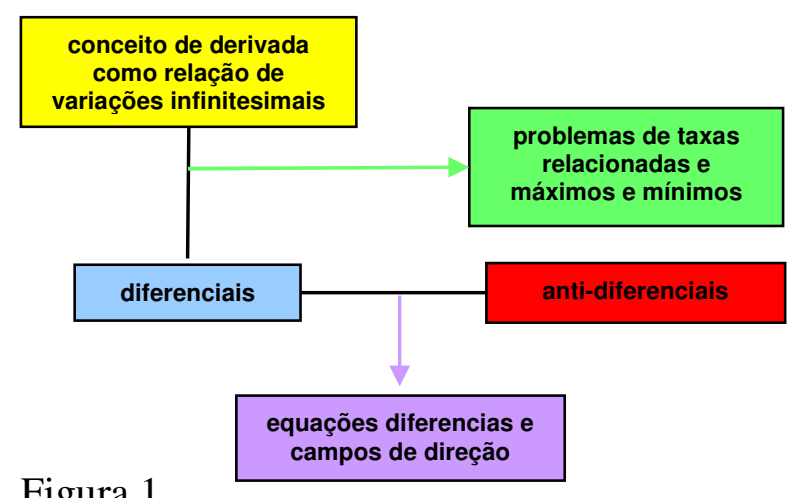
disciplinas tivessem por finalidade o trabalho de fundamentar aplicações. De outro modo, as aplicações devem ser identificadas à formação profissional e a outras disciplinas que formam a rede curricular de formação do engenheiro de sistemas digitais. Essa integração entre disciplinas passa a ser denotada rede de conhecimentos na qual o aluno deve se reconhecer e se integrar. Isto é mais do que conhecimento para o aluno; trata-se de conhecimento do aluno. É ele quem deve deter e trabalhar para organizar seu conhecimento.

A posição epistemológica adotada - legitimidade dos conteúdos na pedagogia e a transposição didática (Chevallard, 1991) - tem permitido a abordagem de modo independente do cálculo de diferenciais das funções elementares pela regra da cadeia e a conceituação da derivada como relação de variações infinitesimais, considerados temas difíceis pelo aluno. Algumas aulas sobre um desses tópicos são seguidas de outras sobre o outro, até que ambos se fundem em problemas de taxas relacionadas e em problemas de máximos de mínimos. A figura 1 é um exemplo de proposta didática amparada nesta epistemologia ${ }^{2}$.

É a visão do "ser engenheiro" que justifica a realização do trabalho didático sobre cálculo diferencial ser suportado pelos infinitésimos ou cálculo infinitesimal; os departamentos de ciência aplicada jamais abandonaram o uso dos elementos infinitesimais. Para o ensino de matemática da engenharia a fundamentação é feita após o primeiro contato do aluno com problemas, sendo suficiente que a instituição através da representação docente garanta que essa fundamentação existe. Assim os alunos podem trabalhar com acréscimos infinitamente pequenos $d x$ da variável $x$ e com deslocamentos infinitamente pequenos $d \overrightarrow{\mathbf{r}}$ de um ponto no espaço, bastando-lhes saber que um infinitésimo é um número hiper-real ${ }^{3}$ cujo valor absoluto é menor que todo numero real positivo.

\footnotetext{
${ }^{2}$ A experiência feita de introduzir o tema equações diferencias na disciplina de Matemática I, no primeiro período letivo do Curso, evoluiu em aplicação na cadeira de Matemática III para modelagem de circuitos elétricos simples RC e RLC.

${ }^{3}$ Os números hiper-reais são definidos como classes de equivalência de seqüências de reais módulo um ultrafiltro livre (Sad, Teixeira e Baldino, 2001). O que a análise não-standard mostra é que podem ser usadas as aproximações por limites, sem que seja necessário dominar a técnica dos épsilons e deltas em nível de graduação. Já foi observado que, não obstante o mérito de alguns textos buscarem a consolidação dos estudos fora do campo da análise real, é preciso reconhecer que mesmo livros como os propostos por Keisler (1986) sobre cálculo diferencial e integral, ainda que concordem quando se argumenta que os infinitésimos estão presentes em nossa intuição (Robinson, 1980; Baldino, 1998), não estão ao alcance da maioria de nossos alunos.
} 


\section{A elaboração de fichas de trabalho e o uso de softwares}

A tecnologia a serviço dos processos de aprendizagem deve permitir desenvolver novas aplicações das disciplinas de matemática (Baldino e Silva, 2001) e produzir a afirmação dos conceitos matemáticos. Para isso, introduzimos objetos matemáticos através de FTs cuja estrutura - problema, encaminhamento solução - é facilmente assimilada pelos alunos de engenharia (Cabral e Baldino, 2004). A idéia é que temas possam ser introduzidos por problemas considerados como aplicações na área de formação. Se um problema apresenta um baixo fator de possibilidade de vir a se constituir uma aplicação direta, lançamos mão do recurso de contextualizar o tema como ferramenta auxiliar nos problemas de engenharia. A exemplo, temos as técnicas de resolução de integrais por partes. Esse tópico passa a ser tratado como ferramenta para o engenheiro que terá de lidar com Transformadas de Laplace (TL) em suas análises de circuitos elétricos ${ }^{4}$, pois a TL de uma função $f(t)$ (que pode ser a modelagem de tensão ou corrente em um circuito) é definida como $\mathcal{L}\{f(t)\}=\int_{0}^{\infty} e^{-s t} f(t) d t$. Visando o sucesso do trabalho didático, são selecionadas funções que recaem em integrais que devem ser resolvidas pelo método de integração por partes. Nesse ponto, não se está pretendendo desenvolver no aluno habilidade com TL, mas, sim, que faça algum sentido para ele a necessidade de aprender uma determinada técnica de integração.

Seguindo a diretriz de manter o aluno engajado na tarefa, cada FT propõe um problema em nível que o desafie, ou seja, nem muito fácil e burocrático, nem muito difícil e desestimulante. A FT traz encaminhamentos, sob forma de sugestões, visando a conduzir o aluno, passo a passo, no rumo da solução esperada. Finalmente, a FT apresenta a solução esperada, estimulando o debate do que foi produzido.

\subsection{Uma ficha de trabalho}

Um dos maiores desafios reportados é levar os alunos a lidar com a regra da cadeia. É uma habilidade importante, principalmente para o aluno de engenharia, que ainda está associada a relatos de dificuldades em cálculo. Cury (2004, 2003a e 2003b), ao trabalhar com análise de erros, relata e interpreta as justificativas criadas pelo aluno ao diferenciar de modo errado uma função composta, ou lidar com derivação. Um exemplo de erro retirado de Cury (2004) e que comumente encontramos entre os alunos é o seguinte: "Dada a função $f(u)=\sin ^{n} u$ o aluno calcula sua derivada da seguinte maneira: $f^{\prime}(u)=n \cos u^{\prime \prime}$.

Para tratar de problemas assim, produzimos uma atividade que introduz as diferenciais ao invés da derivada. Eis o exemplo parcial (figura 2) retirado de uma de nossas FTs para tratar esse problema. A FT é denominada "Diferencias e antidiferenciais". É dada uma tabela de diferenciais que deverá ser consultada pelos alunos enquanto as regras ainda não estiverem disponíveis naturalmente. A tabela traz 19 linhas. Na primeira coluna são apresentadas as funções elementares. A segunda coluna indica a que se deseja diferenciar a função dada, mas chamando atenção para o fato de no espaço preenchido com os pontinhos pode ser inserida uma nova função (função como variável). Finalmente, a terceira coluna apresenta que regra deve ser usada.

\footnotetext{
${ }^{4}$ Nesse sentido, o fato de os professores que se dedicam ao curso estarem sempre juntos e pertencerem a mesma Unidade (ESD) facilita a troca de idéias sobre quais temas introduzir e como fazer para que se firmem como ferramentas para os alunos ao longo de seus estudos. 


\begin{tabular}{|c|c|c|c|}
\hline & Coluna 1 & Coluna 2 & Coluna 3 \\
\hline 0 & $\ldots$ & $d k \ldots=$ & $=k d \ldots$ \\
\hline 1 & $\ldots{ }^{n}$ & $d \ldots{ }^{n}=$ & $=n \ldots(n-1) d \ldots$ \\
\hline 2 & $\sin x$ & $d \sin \ldots=$ & $=\cos \ldots d \ldots$ \\
\hline 3 & $e^{x}$ & $d e \ldots=$ & $=e \ldots d \ldots$ \\
\hline \multicolumn{3}{|c|}{ Figura 2 } \\
\hline
\end{tabular}

A experiência mostra que a regra de uso mais difícil é a da linha zero: diferencial de uma constante. Os alunos são estimulados a verbalizem a regra em uso, então eles lêem a coluna 2 como "a diferencial de uma constante vezes uma coisa é" e a coluna 3 como "é a constante vezes a diferencial da coisa". No lugar da "coisa" poderíamos ter a função que ele encontra na linha 3, por exemplo. Sempre que os alunos encontram os três pontinhos eles aprendem que devem prestar atenção ao uso de uma segunda ou mesmo terceira regra.

Assim sendo, a proposta de produzir um sistema inteligente (Rezende, 2003) visando aulas de matemática está sendo guiada pela concepção de se dispor de acervo de exercícios e técnicas, relatórios de sala de aula e comentários pedagógicos que contemplem a via da concepção dos infinitesimais junto com a da concepção de limites.

\section{Protótipo desenvolvido: E-M@T - ambiente interativo multidisciplinar para engenharia 5}

Para chegar a um protótipo de um ambiente computacional de educação para engenharia foram considerados os seguintes pontos:

- os alunos, de modo geral, apresentam dificuldades para lidar com linguagem e com a sintaxe de aplicativos matemáticos e gráficos,

- com a Internet, a interfaces gráficas de funcionamento em WEB ficaram bem conhecidas e permitem que o trabalho seja realizado de modo direto e simples,

- a organização de exercícios tinha de ter uma estrutura semelhante a de uma FT

- o trabalho didático do professor deveria ser facilitado por uma ferramenta de autoria que o permitisse criar objetos de aprendizagem dentro do próprio sistema.

Foi projetada então uma infra-estrutura computacional, denominada E-M@T, para sala de aula regular visando os processos de ensino e de aprendizagem na engenharia. Essa estrutura consiste em um sistema ativo de disseminação de informações, conteúdos, exercícios e aplicações consideradas necessárias para os processos de ensino e de aprendizagem. Em seu estágio atual, o sistema está estável, tendo passado por testes técnicos, o que possibilita atribuir-lhe a denominação E-M@T - ambiente interativo multidisciplinar de educação para engenharia.

\footnotetext{
${ }^{5}$ A versão 1.0 do E-M@T foi desenvolvido pela equipe inicial do GrITEE - professores: João Carlos Gluz, Roberto Ribeiro Baldino e Tânia C. B. Cabral e alunos: Paulo Baggio, Paulo Livi e Rafael Mallmann. O sistema e demais aplicativos estão disponíveis em www.gritee.com. O grupo hoje é integrado por mais professores e alunos da própria Unidade de Guaíba e professores e alunos das Unidades Caxias do Sul e Novo Hamburgo e um docente da USP. V.3 $\mathrm{N}^{\mathrm{o}} 2$, Novembro, 2005
} 
$\mathrm{Na}$ fase de desenvolvimento em que o projeto está, são três seus pilares, em perfeita sintonia com toda argumentação exposta no trabalho. Um pilar concerne a sua característica técnica que o define como um ambiente baseado na idéia de uso de aplicativos Open Source e projetado para funcionar em rede em aula presencial. Relacionado ao trabalho de ensino e de aprendizagem, temos o segundo pilar que concerne aos aspectos didáticos e epistemológicos já apresentados: as atividades são concebidas na transposição didática que visa estabelecer objetos profissionais buscando sempre o estado da arte técnico e científico. E o terceiro pilar é referente ao aspecto pedagógico, também bastante discutido: é um ambiente que visa promover o trabalho interativo e cooperativo de aprendizagem útil e produtivo. Os objetivos propostos que definem o funcionamento e o papel do E-M@T são: (1) servir como ambiente de trabalho para o ensino e para aprendizagem em sala de aula regular - educação presencial sob supervisão do professor (agente externo), (2) servir com uma ferramenta para os alunos estudarem fora de sala de aula e (3) abranger o maior número possível de conteúdos referentes às disciplinas de um curso de engenharia. Para alcançar esses objetivos, selecionamos aplicativos Open Source, seguindo diretrizes epistemológicas determinantes para o ensino e para a aprendizagem. O E-M@T deve suportar atividades nas mesmas bases de trabalho ao serem incorporadas a transposição didática e a resolução de problemas.

O E-M@T é uma interface gráfica que tem por célula mínima o uso de Ficha de Trabalho Eletrônica (FTE), objetos de aprendizagem, obedecendo aos mesmos critérios de engenharia didática das Fichas de Trabalho (FTs) com que vimos trabalhando em sala de aula de matemática.

\subsection{Arquitetura cliente-servidor}

A concepção de sua arquitetura de funcionamento é simples: cliente-servidor. É preciso que os computadores estejam em rede com um servidor onde o E-M@T fica armazenado. O professor, a partir de seu monitor, autoriza o começo da aula. As atividades projetadas pelo professor são distribuídas pelo servidor para os computadores dos alunos. Os grupos de alunos, diante do computador, trabalham, experimentam e devolvem respostas das atividades ao servidor.

Essa arquitetura permite que possam ser feitas atualizações e manutenções do sistema pelo administrador a qualquer tempo, não necessitando que sejam compiladas e instaladas novas versões individualizadas do sistema. O E-M@T é suportado por navegadores bem conhecidos pelos usuários de Internet como Internet Explorer, Netscape e Firefox; funcionando tanto no SO Windows como no SO Linux. O agente administrador, professor ou aluno - é identificado pelo sistema que o remeterá para o modo de trabalho conforme o login especificado. A seguir, temos as principais características de cada sistema modelado.

\section{a) O Sistema Administrador}

Compete ao administrador, cujos conhecimentos de informática são suficientes para programar, exercer a função de manter o sistema funcionando no servidor. Como a própria denominação deixa indicar, deve cadastrar professores e disciplinas, assegurar que a base de dados esteja em perfeita ordem e segura por backup periódico. Qualquer bug no sistema é imediatamente reportado ao

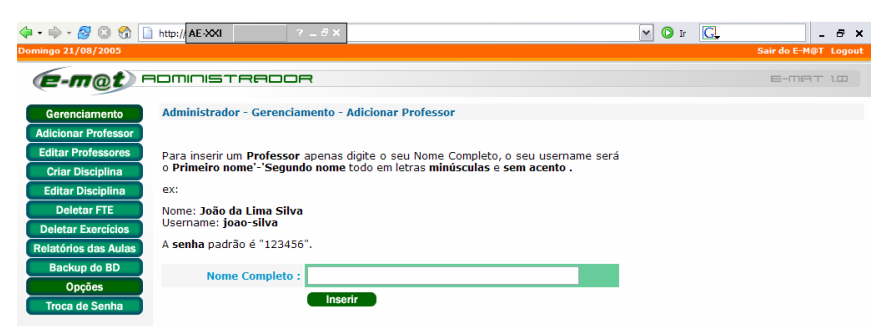


administrador que, conhecendo a modelagem e construção do sistema, terá condições de retificar tais problemas. A tela 1 mostra o cadastramento de um usuário (professor).

\section{b) O Sistema Professor}

O Sistema Professor foi projetado para oferecer opções de trabalho didático e pedagógico como o trabalho com várias turmas sob a regência do mesmo docente, podendo gerar as turmas pelas quais está responsável ao longo do semestre ou ano letivo. Gerada cada turma, o próprio professor edita as listas dos alunos de cada turma. O trabalho de projetar tarefas e atividades na forma de FTEs é independente da turma. Isto é, o professor pode criar uma FTE ou usar uma que já esteja disponível no sistema para trabalhar com qualquer uma das turmas geradas.

Para simplificar a tarefa do professor na criação de uma FTE projetamos uma ferramenta de autoria. Isto é, o professor além de poder produzir e inserir uma FTE no sistema, ele pode criar a FTE no próprio sistema contribuindo para a formação de uma base de dados que estará disponível para uso de qualquer professor cadastrado no sistema. A ferramenta disponível para a criação de FTE é um editor HTML WYSIWYG que permite, inclusive, lidar com fórmulas matemáticas que, ao serem introduzidas no formato string, são lidas e devolvidas em

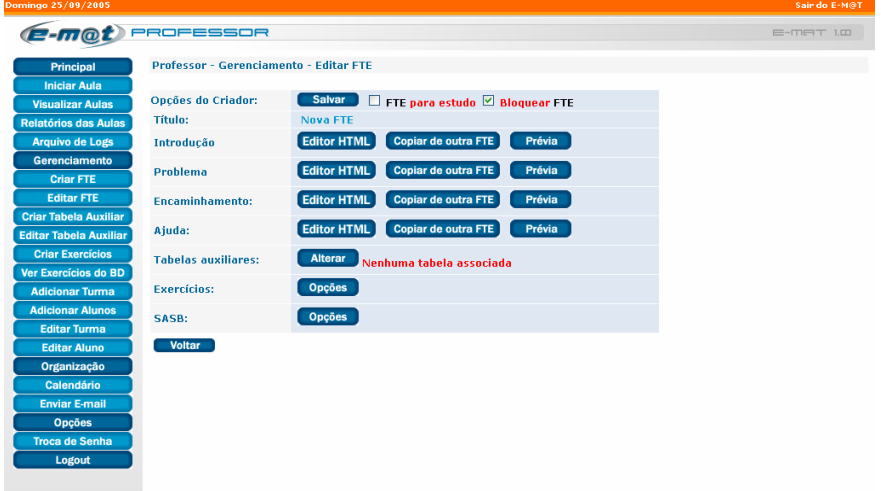
MathML na forma matemática usual. A ferramenta de autoria (tela 2), que está sendo aperfeiçoada, foi criada diante de uma dificuldade que temos visto na utilização de outros ambientes computacionais onde o professor deve saber lidar com linguagem de programação para projetar uma atividade eletronicamente.

Durante uma aula em andamento, o professor dispõe de uma ferramenta que lhe permite visualizar através de seu monitor como cada grupo se movimenta por entre os exercícios propostos. De certa maneira, o professor tem uma informação sobre as dificuldades da atividade que foi proposta, pois, a partir da experiência que o professor acumula e conhecendo as habilidades de seus alunos, é-lhe possível saber se uma atividade está ou não ao alcance de o aluno trabalhar. Se grupos avançam e um grupo permanece na mesma atividade, o professor sabe que é muito pontual a dificuldade que está sendo enfrentada. Enquanto que, se vários grupos permanecem muito tempo no mesmo item, interpretamos que pode ter havido falhas na projeção da atividade, quer seja em termos do problema proposto ou mesmo do encaminhamento.

\section{c) O Sistema Aluno}

O Sistema oferece dois modos de funcionamento para o aluno trabalhar, porém não arbitrário. $\mathrm{O}$ aluno para acessar o E-M@T recebe um nome de usuário e uma senha. O sistema de login, ao identificar o aluno direciona-o ou para o Modo Aula ou para o Modo Estudo, conforme o estado da aula verificada pelo próprio sistema - aula autorizada em andamento ou não, pelo professor.

No Modo Aula, como acontece em qualquer sala de aula regular, o aluno está submetido às condições que o professor estabelece sobre as atividades didáticas escolhidas para o trabalho do dia. Uma vez que a classe é organizada em pequenos grupos e as solicitações dos alunos por atendimento do professor ocorrem ao mesmo 
tempo, foi desenvolvida uma ferramenta que possibilita ao aluno "chamar" o professor em caso de dúvidas ou discussões provocadas pela atividade; os alunos são encorajados a debaterem com outros colegas e o professor o que estão experimentando. O professor tem condições de visualizar as chamadas no monitor de sua estação de trabalho.

No Modo Estudo, entretanto, o aluno tem condições de arbitrar sobre o assunto com o qual trabalhará. Na fase em que estamos, o aluno tem acesso somente às FTEs que já foram trabalhadas em classe regular. Desse modo, funciona mais ou menos como revisão de matéria estudada. $\mathrm{O}$ objetivo que temos para seguir adiante é disponibilizar para o aluno atividades complementares que poderão ser por ele acessadas no Modo Estudo e que não necessariamente foram trabalhadas em aula presencial.

\subsection{SASB: sistema de organização de softwares Open Source}

O desenvolvimento do sistema tem demandado várias frentes de trabalho sob certas condições, todas atendidas sem que haja prejuízo para o desenvolvimento da parte técnica. Uma frente de trabalho se ocupou da projeção da interface gráfica responsável pela apresentação do E-M@T, produzindo um sistema agradável e de fácil utilização para os usuários finais - professores e alunos. Sempre se visa que não deve haver qualquer necessidade de se ter conhecimento específico sobre programação ou equivalente. Outra exigência estabelecida e atendida diz respeito à interferência da interface do E-M@T sobre o trabalho. Conseguiu-se que a apresentação gráfica não desviasse a atenção do aluno sobre o trabalho de aprendizagem de objetos-matemáticos profissionais, finalidade para a qual o ambiente foi inicialmente projetado.

\section{A escolha de softwares}

Para propor um ambiente computacional educacional, o primeiro passo foi buscar um sistema computacional algébrico (CAS - Computer Algebra System) que atendesse a exigência que temos de lidar com funções, cálculos diferenciais, cálculos integrais, gráficos e outros tópicos. Foram avaliados 5 aplicativos (Máxima, Scilab, MuPAD, Functions, YACAS) em função de instrumentos necessários ao cálculo diferencial e integral e principalmente que atendesse ao caráter diferencial.

Apesar de quase todos os aplicativos, do ponto de vista dos conteúdos abordados, terem sido avaliados como possíveis para usar, um deles chamou atenção pela "afinidade" com a proposta de trabalhar os elementos infinitesimais e pela flexibilidade para ter seu código modificado e adaptado diretamente para este fim: o Maxima Primer. Como primeira experiência foi projetada uma FTE associada pela primeira vez a um software que daria apoio ao aluno para trabalhar o método passo a passo de diferenciar uma função composta.

O trabalho foi, e está sendo, procurar e adaptar softwares para funcionar no ambiente montado segundo a estrutura cliente-servidor de modo que sejam acessados remotamente, servindo para o aluno propor, comparar e testar soluções. Essa exigência diz respeito à organização de softwares para funcionar como ferramentas de acesso em backend. Foi construído um sistema de caráter frontend denominado SASB - Sistema de Acesso a Softwares em Backend - para orgi (E-MOT) usuário e máquina. Foram incorporados aplicativı gráficos de funções, o Máxima, para o trabalho trabalhar com matrizes e equações diferencias. A auxiliou na plotagem de um gráfico.

Outro ponto importante para o desenvolvimento símbolos matemáticos serem visualizados nas sua 10

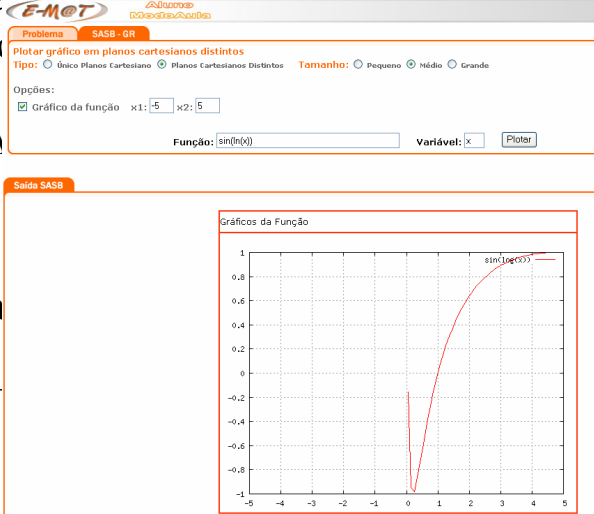


indefinida da função seno de variável $x$, por exemplo, deveria aparecer como $\int \sin x d x$ e não apenas na forma de string $\operatorname{int}(\sin (x), x)$, não obstante terem sido projetadas atividades para introduzir e familiarizar o aluno com essa forma de escrita, uma vez que o uso de calculadoras gráficas e as planilhas eletrônicas demandam familiaridade com essa forma. Para retornar na tela a forma matemática usual foi preciso uso do MathML.

\section{Considerações}

O E-M@T foi usado em aulas regulares de Matemática 1 de modo experimental, visando também testes para estabilizar o sistema. Os alunos que lidaram com o ambiente, de modo informal, avaliaram que haver um sistema sendo desenvolvido na própria instituição é uma idéia boa e pertinente ao curso. Com relação ao trabalho visando a aprendizagem com diferenciais, o resultado pode ser considerado positivo: os alunos, por terem de fazer comparações entre o que propuseram como resposta e o que o sistema devolvia como solução, tiveram de estabelecer perguntas inclusive sobre o comportamento de certas funções. $\mathrm{O}$ fator curiosidade esteve presente durante a aula.

Além disso, o sistema por ser voltado para disciplinas de Matemática, que em outros cursos são dadas de modo que não têm ligação com as formações específicas, está sendo considerado um diferenciador. Tem aumentado o interesse de alunos para usar o sistema mesmo que seja em modo remoto, a partir de suas casas. Em função dessa demanda estamos investindo trabalho de pesquisa para gerar um protótipo que atenda, nesse caso, a educação a distância destinada a área técnica, baseado na idéia de interatividade.

Outro ponto positivo é que os alunos reconhecem o E-M@T como uma produção de um grupo de colegas e professores dedicados ao curso de engenharia que buscam inovar em aspectos que possam contribuir para a formação global do futuro engenheiro. Tal quadro tem se mostrado importante, pois está havendo engajamento de alunos, mesmo como voluntários, em projetos na instituição. Há um forte apelo para tomar parte do desenvolvimento de ferramentas computacionais e softwares que possam ser usados através da administração de ambientes como o E-M@T destinado a educação em engenharia.

Os estudos realizados e as concepções que tivemos de especificar, considerada a demanda por uso de computadores em aula presencial, a leitura de bibliografia especializada no campo da educação matemática e na educação em engenharia, são fontes de orientação precisa e consistente na construção e desenvolvimento do ambiente. Têm proporcionado, sobretudo, informações para a modificação da própria sala de aula. Ademais, sem a clareza sobre as condições psicológicas de aprendizagem, conforme bastante argumentado neste trabalho, não teria sido possível a construção do E-M@T. Em concordância com autores citados, sem analisar os componentes psicológicos e sociais não se tem como propor modificações das condições de ensino e de aprendizagem e muito menos propor a inserção em sala de aula de um apoio baseado em tecnologia. Ao mesmo tempo em que se propõe inovar em termos tecnológicos é preciso reconsiderar a própria organização de conteúdos com que se lida e sua relação com temas e conceitos de outras disciplinas que fazem parte do mesmo grupo de formação de um profissional.

Mas é preciso também dizer que ainda há muito trabalho a ser feito com relação a formulação de FTEs que possam integrar o sistema aqui apresentado. Nesse ponto, a dedicação ao funcionamento técnico e estabilização do sistema, consome parte de nossos esforços. Sem contar que outros colegas, professores, sensibilizados pelos 
aspectos da educação em engenharia, que tomam parte do grupo de pesquisa, estão mergulhados no trabalho de (1) agregar inteligência ao sistema visando atender a demanda de avaliação e (2) contribuir com novas funções para o SASB e (3) ampliar um banco de atividades compartilhadas. Não seria nada complicado se não fosse o fato de termos definido como proposta de avaliação a idéia de fazer encaminhamentos, sob forma de sugestões, visando a conduzir o aluno, passo a passo, no rumo da solução esperada. Não se quer dar ao aluno o veredicto final sobre sua resposta: está certo ou está errado. Em consonância com a discussão feita e que seguimos em aula, procuramos colocar o aluno em situação de ele próprio, amparado por sugestões, questões ou situações de conflito, poder avaliar suas respostas.

A diretriz é acenar com a possibilidade de dar tratamento a certos problemas de não crescimento cognitivo ao se fazer uso da instauração de conjunto de imagens onde o objeto-matemático profissional possa ser construído. No caso do curso de engenharia de sistemas digitais, reforçamos a idéia que o uso de ambientes computacionais podem fazem parte desse leque como indicamos a seguir, ao apresentar o que recebemos de opiniões, avaliações e sugestões feitas pelos alunos.

Atualmente, professores das Unidades de Caxias do Sul e Novo Hamburgo da UERGS, junto com seus orientados, estão trabalhando para levar para seus cursos de engenharia o ambiente E-M@T e estão tomando parte nos trabalhos de pesquisa que tem por objetivo reforçar o caráter multidisciplinar do ambiente ao projetarem softwares e atividades.

Em relação a desenvolvimento do ambiente, em longo prazo, pode-se prever a incorporação de um nível de inteligência no sistema que deverá refletir: (1) outra forma de apresentação dos conteúdos, (2) flexibilidade e maior interação entre usuários e máquina, (3) avaliação da produtividade dos alunos e (4) a possibilidade de utilização do sistema como instrumento voltado para educação a distância. Em qualquer caso, existem várias questões que requerem, no mínimo, a incorporação da função do usuário, quer seja aluno, professor ou monitor, no processo de suporte do sistema. Pela experiência relatada em projetos de ambientes inteligentes no setor produtivo (Rezende, 2003) e no setor de ensino (Seixas et al. 2002; Flores et al. 2003) e no uso de sistemas tutores inteligentes (Girafa, Mora e Viccari, 1999), pode-se antever que para se adicionar inteligência ao sistema, deverá ser necessário também agregar semântica ao processamento dos objetos de aprendizagem, as FTEs, de forma que sejam capazes de "compreender" o assunto (ou parte dele) que está sendo ensinado.

Em resumo, queremos continuar no caminho de realizar uma proposta multidisciplinar, através de ações propostas e desencadeadas pelo projeto.

\section{Agradecimentos e créditos}

Meus agradecimentos são dirigidos aos colegas e alunos que compuseram a equipe inicial do GrITEE, pois juntos trabalhamos na criação do E-M@T. São meus colegas, professores do Curso de Engenharia em Sistemas Digitais, João Carlos Gluz e Roberto Ribeiro Baldino. São nossos alunos de iniciação científica, responsáveis pela programação e execução técnica do sistema na versão 1.0: Paulo Baggio, Paulo Livi e Rafael Mallmann. Agradeço ao Coordenador da Unidade, Professor Gilson Wirth, e ao Pró-reitor de Pesquisa, Professor Gerhard Jacob, pelo apoio institucional. Agradeço à FAPERGS e ao CNPq por estarem apoiando com bolsas este projeto em sua nova fase. Hoje o GrITEE, através do projeto, congrega mais docentes e discentes de outras Unidades da UERGS.

\section{Referências}


ALLEVATO, N. S. G. (2003). A associação dos computadores à metodologia de resolução de problemas trazendo novas possibilidades para o ensino de Matemática. In Anais da $11^{\text {th }}$ Inter-American Conference on Mathematics Education, july 13-17, 2003. Blumenau (SC): FURB. CD-R.

AZAMBUJA, C. R. J. de, SILVEIRA, F. A. R. e GONÇALVES, N. da S. (2004). Tecnologias síncronas e assíncronas no ensino de cálculo diferencial e integral. In Cury, H. (organizadora), Disciplinas matemáticas em cursos superiores: reflexões, relatos e propostas. Porto Alegre: EDIPUCRS, 2004.

BALDINO, R. R. (1998). Desenvolvimento de Essências de Cálculo Infinitesimal. Rio de Janeiro: MEM/USU.

BALDINO, R. R. (1991). A Interdisciplinaridade da Educação Matemática. Didática, Vol. 26/27, 1990/1991, p. 109-121. Editora da UNESP.

BALDINO, R. R. \& SILVA, R. H. da (2001). Introdução ao processamento de imagens ou aplicação da álgebra linear? Revista de Matemática e Estatística, V. 19, p. 123144. São Paulo: Editora da UNESP. (25/06/98)

BORBA, M. C. (2005). The transformation of mathematics in on-line courses. In Chick, H. and Vincent, J. (editors), Proceedings of the $29^{\text {th }}$ Conference of the International Group for the Psychology of Mathematics Education, v.2, p. 169-176. Melbourne: PME, 2005.

BORBA, M. C. and VILLARREAL, M. E. (2005). Humans-with-media and the reorganization of mathematical thinking: information and communication technologies, modeling, experimentation and visualization. EUA: Springer.

CABRAL, T. C. B. (1998). Contribuições da Psicanálise à Educação Matemática: a lógica da intervenção em processos de aprendizagem. Tese de Doutorado. São Paulo: USP.

CABRAL, T. C. B. e BALDINO, R. R. (2004). O ensino de matemática em um curso de Engenharia de Sistemas Digitais. In CURY, H. (organizadora), Disciplinas matemáticas em cursos superiores: reflexões, relatos e propostas. Porto Alegre: EDIPUCRS, 2004.

CAMARGO Jr., João Batista, CUGNASCA, Paulo Sérgio e ALMEIDA JR., Jorge Rady (2003). O treinamento de docentes para o ensino cooperativo de engenharia foco no aprendizado do aluno. In Anais do XXXI COBENGE, 2003. CD-ROM.

CARDOSO, Rodrigo Ferrugem (2001). AvalWeb - Sistema interativo para gerência de questões e aplicação de avaliações na Web. Porto Alegre, RS: PPGC da UFRGS, 2001.

CHEVALLARD, Y. (1991). La transposición didáctica: del saber sabio al saber enseñado. Buenos Aires: Aique.

COSTA, L. A. C. da (2004). A avaliação da aprendizagem no ensino de estruturas: epistemologia, tecnologia e educação à distância. Tese (doutorado), Universidade Federal do Rio Grande do Sul. Escola de Engenharia. Programa de Pós-Graduação em Engenharia Civil. Porto Alegre, BR, RS, 2004.

CURY, H. N. (2004). Da saliva e do pó de giz ao software de computação algébrica: a difícil adapatação dos profesores de matemática às exigencias da sociedade informatizada. In Cury, H. (organizadora), Disciplinas matemáticas em cursos superiores: reflexões, relatos e propostas. Porto Alegre: EDIPUCRS, 2004.

(2003 a). Análise de erros e análise de conteúdo: subsídios para uma proposta metodológica. In Anais do II SIPEM, 2003. CD-ROM.

(2003 b). Análise de erros em cálculo diferencial e integral; resultados de investigações em cursos de engenharia. In Anais do XXXI COBENGE, 2003. CDROM. 
FERREIRA, André Luis Andrejew (1999). Avaliação de software no ensino de cálculo. Porto Alegre, RS: PPGC da UFRGS, 1999.

FLEMMING, D. M. (2004). O ensino de cálculo nas engenharias: relato de uma caminhada. In CURY, H. (organizadora) Disciplinas matemáticas em cursos superiores: reflexões, relatos e propostas. Porto Alegre: EDIPUCRS, 2004.

I FLORES, C.; GLUZ, J.; SEIXAS, L.; VICCARI, R.; COELHO, H. Pedagogical Negotiation in AMPLIA Environment. In: Proceedings of Argentine Symposium on Artificial Inteligence, Buenos Aires, 2003.

GARCIA, A. C., VAREJÃO, F. M. e FERRAZ, I. N. (2003). Aquisição de conhecimento. In REZENDE, S. O. (coord.) (2003). Sistemas inteligentes: fundamentos e aplicações. Barueri, SP: Manole, 2003.

II GIRAFFA, L. M. M.; MORA, M.; VICCARI, R. M. (1999). Towards a New Perspective for ITS: Using a Mentalistic Approach to Model Cognitive Pedagogical Agents. Special Issue on Intelligent Systems/Tools in Training and life-Long Learning. International Journal of Continuing Engineering Education, Osaka, Japão, 1999.

GONÇALVES, Eliete M., CHUERI, Vanilda M. M. e SARCOMAN, Marco A. R. (2002). Uma ferramenta computacional para o ensino de funções nos cursos de engenharia. In Anais do XXX COBENGE. Piracicaba, SP: UNIMEP, 2002. CDROM.

KEISLER, H. J. (1986). Elementary Calculus: an infinitesimal approach. Boston: PWS Pub.

LINS, Bibi, GIRALDI, Victor et al. (2004). Toward new perspectives and new methodologies for the use of technology in mathematics education. In Høines, Marit and Fuglestad, Anne Berit (editoras), Proceedings of the $28^{\text {th }}$ Conference of the International Group for the Psychology of Mathematics Education, v. 1, p. 265, Bergen, No: Bergen University College, 2004.

LODER, L. L. (2001). Epistemologia versus pedagogia: o locus do professor de engenharia. Anais do XXIX COBENGE, Porto Alegre: PUC 2001. CD-ROM.

MENDES FILHO, L. A. M. (2001). et al. Inovações tecnológicas no ensino: contribuições teóricas. Anais do XXIX COBENGE, Porto Alegre: PUCRS, 2001. CD-ROM.

PICHON-RIVIÈRE, Enrique (1988). O processo grupal. São Paulo: Martins Fontes.

PROJETO E-M@T (2005): Acessível em www.gritee.com.

REZENDE, S. O. (coord.) (2003). Sistemas inteligentes: fundamentos e aplicações. Barueri, SP: Manole, 2003.

ROBINSON, A. (1966). Non-Standard Analysis. Amsterdam: North Holland.

SAD, L. A., TEIXEIRA, M. V. e BALDINO, R. R. (2002). Cauchy and the problem of point-wise convergence. Archives Internationales D'histoire Des Sciences, Liège, Bélgica, v. 52, n. 148, p. 1-32. (16, 17, 18/03/2002)

SEIXAS, L.; FLORES, C.; VICCARI, R.; LADEIRA, M. (2002). An Architecture for an Intelligent Learning Environment with a constructivist approach. In: Workshop Architectures and Methodologies for Building Agent-based Learning Environments. ITS2002. San Sebastian, Spain, 2002.

VIALI, L. (2004). Utilizando recursos computacionais (planilhas) no ensino de cálculo de probabilidades. In Cury, H. N. (organizadora), Disciplinas matemáticas em cursos superiores: reflexões, relatos e propostas. Porto Alegre: EDIPUCRS, 2004. 